\title{
Targeting dihydroorotate dehydrogenase in acute myeloid leukemia
}

\author{
Zhihong Zeng $^{1}$ and Marina Konopleva ${ }^{1,2}$
}

\author{
${ }^{1}$ Department of Leukemia and ${ }^{2}$ Department of Stem Cell Transplantation and Cellular Therapy, The University of Texas MD Anderson \\ Cancer Center, Houston, TX, USA
}

E-mail: mkonople@mdanderson.org

doi:10.3324/haematol.2018.197806

W ith their discovery of a promising inhibitor of dihydroorotate dehydrogenase (DHODH), Wu and Wong et al. have made progress toward effective differentiation therapy for acute myeloid leukemia (AML). ${ }^{1}$ Their paper entitled "Pharmacological inhibition of dihydroorotate dehydrogenase induces apoptosis and differentiation of acute myeloid leukemia cells," published in this issue of Haematologica, reports that this inhibitor had significant anti-leukemia efficacy in vitro and in vivo. ${ }^{1}$

$\mathrm{DHODH}$ is an enzyme that catalyzes the oxidation of dihydroorotate into orotate in intracellular de novo pyrimidine synthesis. Pyrimidine bases are essential for cellular metabolism and cell growth, and are considered important precursors in nucleotide, glycoprotein, and phospholipid biosynthesis and nucleotide recycling. ${ }^{2}$ Dysregulation of and functional dependency on pyrimidine biosynthesis have been found in diverse solid tumors. ${ }^{3}$ As a key enzyme regulating this process, DHODH has been identified as a synthetically lethal target in tumors that carry specific genetic mutations, including PTEN-deficient and triple-negative breast cancer, ${ }^{4,5}$ BRAF ${ }^{\mathrm{V} 600 \mathrm{E}}$-mutant melanoma, ${ }^{6}$ RAS/LKB1 double-mutant lung cancer, ${ }^{7}$ and KRAS-mutated pancreatic cancer. $^{8}$

AML is a highly heterogeneous hematologic malignancy, characterized by uncontrolled growth of immature hematopoietic stem/progenitor cells and impaired differentiation. ${ }^{9,10}$ The long-term survival rate of AML patients who receive standard therapies remains poor. ${ }^{11}$ Differentiation therapy was proposed decades ago with the goal of promoting the normal process of hematopoietic maturation from self-renewing progenitors to terminally differentiated effector cells, ${ }^{12}$ but its success has been limited except in specific genetic subtypes of AML. For example, all-trans retinoic acid induces differentiation and remissions in patients with acute promyelocytic leukemia. ${ }^{13}$ Inhibitors of isocitrate dehydrogenase (IDH) have been approved more recently for treatment of AML carrying IDH1/2 mutations, and the activity of these inhibitors is associated with clinical and morphological signs of myeloid differentiation. ${ }^{14}$

Recent pre-clinical findings by Sykes et al. showed that $\mathrm{DHODH}$ is a metabolic regulator in the pyrimidine synthesis pathway and a new metabolic target in differentiation therapy for AML. ${ }^{15}$ Inhibition of DHODH effectively enabled cell differentiation and had anti-leukemia efficacy in vitro and in vivo. ${ }^{15}$ This discovery opens up a new perspective in differentiation therapy for AML carrying complex and heterogeneous combinations of gene mutations. It leads to pharmacological intervention via development of novel, potent, and optimized DHODH inhibitors for clinical applications and to studies aiming to determine the mechanisms underlying DHODH inhibition-mediated AML differentiation.

In this issue of Haematologica, Wu and Wang et al. confirm the essential role of DHODH in AML survival and differen- tiation. ${ }^{1}$ Using CRISPR-Cas9-mediated gene knockout, the authors observed induction of apoptosis and cellular differentiation in DHODH-knockout AML cells. Through analysis of RNA sequencing data for 173 primary AML samples, they found that DHODH and MYC, a key regulator of myeloid cell proliferation and differentiation, are coordinately expressed in AML. Using structure-based virtual screening of 337 natural products, the research team identified isobavachalcone, a chalcone derived from the traditional Chinese medicine Psoralea corylifolia, as a potent and selective inhibitor of $\mathrm{DHODH}$. Isobavachalcone, a competitive inhibitor of $\mathrm{CoO}_{0}$ and a noncompetitive inhibitor of the DHODH substrate dihydroorotate, binds the docking site of the "ubiquinone channel" of the DHODH complex, interacting structurally with and stabilizing DHODH. Treatment with isobavachalcone led to dose-dependent induction of apoptosis and myeloid differentiation in the cell lines tested. The on-target effect mediated by isobavachalcone's inhibition of DHODH is supported by the findings that uridine, a downstream metabolite of DHODH in the pyrimidine synthesis pathway, was depleted in isobavachalcone-treated cells and that growth inhibition was rescued by uridine supplementation. ${ }^{1}$ Mechanistically, the apoptosis and cell differentiation induced by isobavachalcone's inhibition of DHODH was found to occur, at least in part, through cMYC suppression, by directly reducing its gene transcription and degrading c-MYC protein via proteasome dependent degradation and downregulation of the enzyme O-linked Nacetylglucosamine transferase that normally stabilizes MYC via transfer of GlcNAc from uridine diphosphate-GlcNAc to MYC. ${ }^{16,17}$ Notably, O-GlcNAc post-translational modification similarly stabilizes additional proteins such as AKT and the TET family of proteins. ${ }^{18}$ In a subcutaneous AML cell line xenograft model, isobavachalcone treatment suppressed tumor growth, and this effect was accompanied by inhibition of DHODH activity and induction of apoptosis of tumor cells. ${ }^{1}$ Oral administration of isobavachalcone was well tolerated in mice. A combination of isobavachalcone and adriamycin (doxorubicin), an agent widely used in AML therapy, synergistically induced cell death and restored sensitivity of doxorubicin-resistant AML cells in vitro; this combination also led to cell differentiation and prolonged mouse survival in vivo in a systemic AML cell line xenograft leukemia model, analogous to the report of the efficacy of doxorubicin combined with the approved DHODH inhibitor leflunomide in triple-negative breast cancer. ${ }^{5}$ These data support the idea that isobavachalcone, an orally active DHODH inhibitor, might have clinical benefit in AML differentiation therapy.

The work by Wu and Wang et al. advances our knowledge of DHODH regulation in AML and offers a novel inhibitor with promise for differentiation therapy. The detailed mechanisms underlying this activity, such as how the functional 


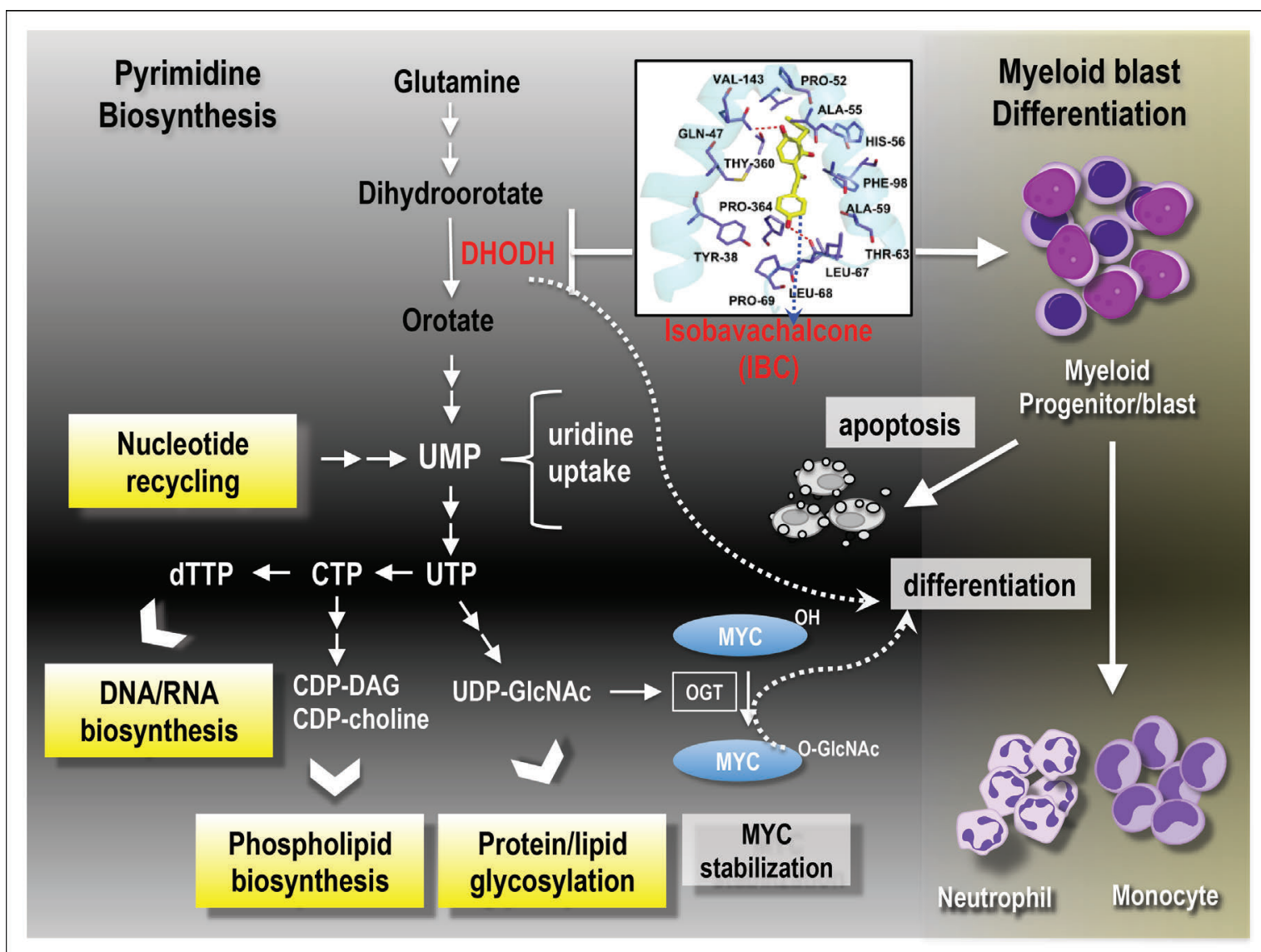

Figure 1. Isobavachalcone in the regulation of dihydroorotate dehydrogenase in pyrimidine biosynthesis and myeloid blast differentiation. Left, the pyrimidine biosynthesis pathway and its biological functions. Isobavachalcone (IBC) prevents DHODH from catalyzing dihydroorotate into orotate, blocking pyrimidine biosynthesis, resulting in myeloid lineage differentiation and induction of apoptosis. The role of DHODH and the negative impact of c-MYC on myeloid blast differentiation is indicated by the dotted line/arrow. DHODH: dihydroorotate dehydrogenase; UMP: uridine 5'-monophosphate; UTP: uridine triphosphate; CTP: cytidine triphosphate; dTTP: deoxythymidine triphosphate; UDP-GlcNAc: uridine diphosphate N-acetylglucosamine; CDP-DAG: cytidine diphosphate diacylglycerol; CDP-choline: cytidine diphosphate choline; O-GIcNAc: O-linked N-acetylglucosamine; OGT: O-GIcNAc transferase. CTP to dTTP requires multiple steps.

interplay between DHODH and c-MYC in AML triggers differentiation and whether this is dependent on cellular context, is worthy of future exploration. Importantly, the capacity of isobavachalcone to facilitate blast differentiation in patient-derived xenografts and AML patients with different cytogenetic abnormalities needs to be addressed. In studies similar to that of $\mathrm{Wu}$ and Wong et al., two groups recently reported new findings related to anti-leukemia mechanisms of DHODH inhibition in different types of hematologic malignancies. $\mathrm{DHODH}$ blockade by the chiral tetrahydroindazole ([R]-HZ00), a newly developed DHODH inhibitor, increased p53 activation and enhanced the anti-leukemic effect of the MDM2 inhibitor, Nutlin3a, in p53-WT chronic myeloid leukemia. ${ }^{19}$ PTC299, identified as a VEGFA inhibitor, targeted DHODH, resulting in cell growth inhibition and differentiation in leukemias, including AML, linking $\mathrm{DHODH}$ regulation and stress-induced VEGFA and angiogenesis. ${ }^{20}$ Notably, these studies demonstrated that $\mathrm{DHODH}$ inhibition alone is not sufficient to eliminate leukemia, requiring combination with standard chemotherapeutic agents or target-specific inhibitors. Given the genetic complexity of AML, future use of $\mathrm{DHODH}$ inhibitors in combination with selected drugs with non-overlapping mechanisms of action may tackle specific aspects of the complex pathogenesis of AML and ultimately improve patients' outcomes.

\section{References}

1. Wu D, Wang W, Chen W, et al. Pharmacological inhibition of dihydroorotate dehydrogenase induces apoptosis and differentiation of acute myeloid leukemia cells. Haematologica. 2018;103(9):1472-1483

2. Levine RL, Hoogenraad NJ, Kretchmer N. A review: biological and clinical aspects of pyrimidine metabolism. Pediatr Res. 1974;8 (7):724-734.

3. DeBerardinis RJ, Lum JJ, Hatzivassiliou G, Thompson CB. The biology of cancer: metabolic reprogramming fuels cell growth and proliferation. Cell Metab. 2008;7:11-20.

4. Mathur D, Stratikopoulos E, Ozturk S, et al. PTEN regulates glutamine flux to pyrimidine synthesis and sensitivity to dihydroorotate dehydrogenase inhibition. Cancer Discov. 2017;7(4):380-390.

5. Brown KK, Spinelli JB, Asara JM, Toker A. Adaptive reprogramming of de novo pyrimidine synthesis is a metabolic vulnerability in triple-negative breast cancer. Cancer Discov. 2017;7(4):391-399.

6. Thomas NE, Berwick M, Cordeiro-Stone M. Could BRAF mutations in melanocytic lesions arise from DNA damage induced by ultraviolet radiation? J Invest Dermatol. 2006;126(8):1693-1696.

7. Kim J, McMillan E, Kim HS, et al. XPO1-dependent nuclear export is a druggable vulnerability in KRAS-mutant lung cancer. Nature 2016:538(7623):114-117.

8. Koundinya M, Sudhalter J, Courjaud A, et al. Dependence on the pyrimidine biosynthetic enzyme DHODH is a synthetic lethal vulnerability in mutant KRAS-Driven cancers. Cell Chem Bio. 2018;25(6):705717. 
9. Papaemmanuil E, Gerstung M, Bullinger L, et al. Genomic classification and prognosis in acute myeloid leukemia. N Engl J Med. 2016;374 (23):2209-2221.

10. Saultz JN, Garzon R. Acute myeloid leukemia: a concise review. J Clin Med 2016:5(3)

11. Dombret H, Gardin C. An update of current treatments for adult acute myeloid leukemia. Blood 2016;127(1):53-61.

12. Nowak D, Stewart D, Koeffler HP. Differentiation therapy of leukemia: 3 decades of development. Blood 2009;113(16):3655-3665.

13. Sanz MA, Grimwade D, Tallman MS, et al. Management of acute promyelocytic leukemia: recommendations from an expert panel on behalf of the European LeukemiaNet. Blood 2009;113(3):1875-1891

14. DiNardo CD, Stein EM, de Botton S, et al. Durable remissions with ivosidenib in IDH1-mutated relapsed or refractory AML. N Engl J Med. 2018;378(25):2386-2398
15. Sykes DB, Kfoury YS, Mercier FE, et al. Inhibition of dihydroorotate dehydrogenase overcomes differentiation blockade in acute myeloid leukemia. Cell 2016;167(1):171-186.

16. Dang CV. MYC on the path to cancer. Cell. 2012;149(1):22-35.

17. Itkonen HM, Minner S, Guldvik IJ, et al. O-GlcNAc transferase integrates metabolic pathways to regulate the stability of c-MYC in human prostate cancer cells. Cancer Res. 2013;73(16):5277-5287.

18. Jozwiak P, Forma E, Brys M, Krzeslak A. O-GlcNAcylation and metabolic reprograming in cancer. Front Endocrinol (Lausanne) 2014;5:145.

19. Ladds $M$, van Leeuwen IMM, Drummond CJ, et al. A DHODH inhibitor increases p53 synthesis and enhances tumor cell killing by p53 degradation blockage. Nat Commun. 2018;9(1):1107.

20. Cao L, Branstrom A, Baird J, et al. PTC299 is a novel DHODH inhibitor that modulates VEGFA mRNA translation and inhibits proliferation of a broad range of leukemia cells. Blood. 2017;130(Suppl 1):1371.

\section{The complexity of stem cell transplants: can we improve our understanding?}

\section{Andrea Bacigalupo ${ }^{1}$ and Francesca Bonifazi ${ }^{2}$}

'Fondazione Policlinico Universitario Gemelli IRCCS, Roma and 'Istituto di Ematologia "Seràgnoli", Azienda Ospedaliera Universitaria Sant'Orsola, Bologna, Italy

E-mail:apbacigalupo@yahoo.com

doi:10.3324/haematol.2018.198010

C ox regression analysis can be considered a robust, easy and universal way to calculate the role of variables on outcome endpoints, such as survival, disease-free survival, and so on. The Cox model is a semiparametric approach based on the strong assumption that the effects of different variables on survival (or on the particular endpoint) are constant over time and are additive in a particular scale.

The setting of allogeneic stem cell transplantation is, however, complicated by two additional levels that limit the application of Cox analysis and call for new, more complex, statistical methods: the first is that some variables in allogeneic stem cell transplantation are not timefixed covariates (such as age, gender, or type of donor) but develop after a certain interval of time from transplantation, and need to be accounted for as time-dependent. In other words, with a starting population of patients, some will develop an event (e.g., cytomegalovirus infection) and some will not: a comparison of patients with and without cytomegalovirus infection will need to consider the infection as a time-dependent variable.

A further level of complexity is provided by competing events: a competing event is one that precludes the event of interest from occurring, or significantly changes its probability. Death before cytomegalovirus infection, is a clear example of a competing event for cytomegalovirus infection. Relapse and non-relapse mortality is another clear example of competing events.

So, there are time-fixed covariates, time-dependent events, and competing events.

In a study published in this issue of Haematologica, Fuerst and colleagues have added a fourth level of complexity: they hypothesized that the effect of different covariates may be different at different intervals from transplantation, and this is exactly what they found.

One example is the stem cell source: bone marrow and peripheral blood as sources of stem cells have been compared in numerous prospective and retrospective studies, including meta-analyses, to define which is better, and results have often been conflicting. Again the complexity of transplantation does not make comparisons easy: in the first randomized study ${ }^{2}$ of patients with low-risk disease, receiving a myeloablative regimen and HLA identical sibling grafts, the hazard risk (HR) of death was 1.20 for recipients of peripheral blood compared to bone marrow $(P=0.2)$. In a more recent prospective study ${ }^{3}$ with unrelated donor grafts, using both myeloablative and reduced intensity conditioning regimens for patients with low, intermediate and high-risk disease, the risk of death was 1.20 for bone marrow versus peripheral blood $(P=0.2)$.

Fuerst and colleagues offer a new way of looking into this particular issue: they found that peripheral blood has a significant protective effect on non-relapse mortality early after transplantation, and a significant detrimental effect later on. ${ }^{1}$ The time point for a change of effect on non-relapse mortality was set at 8 months: this means that patients receiving peripheral blood grafts had a lower non-relapse mortality within 8 months (HR: 0.75) and a higher non-relapse mortality beyond 8 months after transplantation (HR:1.38), which were both highly significant effects (Figure 1). There was no protective effect of peripheral blood on relapse, which is the competing event (Figure 1). The authors also looked at a second model of competing events (transplant-related mortality and non-transplant related, or death due to other causes, including relapses), disproving common beliefs; they found no protective effect of peripheral blood as compared to bone marrow grafts on deaths due to other causes, which raises the question of whether peripheral blood should remain the preferred stem cell source in allogeneic stem cell transplants. Indeed an increased risk of chronic graft-versus-host disease seems not to be compensated by 